\title{
DIFFERENTIAL STRUCTURE OF THE THOMPSON COMPONENTS OF SELFADJOINT OPERATORS
}

\author{
GUILLERMINA FONGI AND ALEJANDRA MAESTRIPIERI
}

(Communicated by Joseph A. Ball)

\begin{abstract}
Different equivalence relations are defined in the set $L(\mathcal{H})^{s}$ of selfadjoint operators of a Hilbert space $\mathcal{H}$ in order to extend a very well known relation in the cone of positive operators. As in the positive case, for $a \in L(\mathcal{H})^{s}$ the equivalence class $C_{a}$ admits a differential structure, which is compatible with a complete metric defined on $C_{a}$. This metric coincides with the Thompson metric when $a$ is positive.
\end{abstract}

\section{INTRODUCTION}

Let $L(\mathcal{H})$ be the algebra of bounded linear operators of a Hilbert space $\mathcal{H}$ and $L(\mathcal{H})^{+}$the cone of positive operators. Consider the partial order $\leq$induced in $L(\mathcal{H})$ by $L(\mathcal{H})^{+}$. There is a natural equivalence relation in $L(\mathcal{H})^{+}$which arises from this order (namely, given $a, b \in L(\mathcal{H})^{+}, a \sim b$ if and only if there exist positive numbers $\alpha, \beta$ such that $a \leq \alpha b$ and $b \leq \beta a)$. Each class or Thompson component admits a complete metric, known as the Thompson part metric. In fact, this construction can be done in every closed convex cone of a Banach space. The reader is referred to [10] and [11] to find definitions, properties and applications of this metric.

On the other hand, the set $G L(\mathcal{H})^{s}$ of invertible selfadjoint operators admits a differential structure as a homogeneous space of the group $G L(\mathcal{H})$ of invertible operators; see [5]. The orbit of the identity is the set $G L(\mathcal{H})^{+}$of invertible positive operators, and it is also the Thompson component of every $a \in G L(\mathcal{H})^{+}$. More generally, the components of positive operators are parameterized by the ranges of their square roots; see [2] and [3]. Also, in [2] and [3] the differential and metrical properties of the component of a positive operator are studied in detail. The aim of this paper is to extend this equivalence relation to the set $L(\mathcal{H})^{s}$ of selfadjoint operators and to define a metric on each component that coincides with the Thompson metric in the positive case, also to study the geometrical structure of the components, and finally, to compare both approaches.

The contents of the paper are the following: In Section 2 we recall some classical results about decompositions of selfadjoint operators which are used throughout the paper. Also, the definition and some properties of the Thompson part metric for positive operators are stated. Section 3 contains a brief description of the

Received by the editors December 4, 2006.

2000 Mathematics Subject Classification. Primary 47B15; Secondary 58B20.

Key words and phrases. Selfadjoint operators, Thompson part metric, differential geometry. 
homogeneous structure of $G L(\mathcal{H})^{s}$. We give a new characterization of the orbit of an invertible selfadjoint operator by means of its polar decomposition. In Section 4 we define three equivalence relations on $L(\mathcal{H})^{s}$ that extend the one defined in the cone $L(\mathcal{H})^{+}$and characterize the corresponding equivalence classes, $C_{a}, C_{a}^{1}, C_{a}^{2}$. In Section 5, we show that $C_{a}$ is homeomorphic to the product of two Thompson components of positive operators. This result allows us to define the Thompson metric $d_{T}$ on the component of a selfadjoint operator. It is proved that, for $a \in$ $L(\mathcal{H})^{s}, C_{a}$ admits a natural structure of homogeneous space of an appropriated group and given $b, c \in C_{a}$ there exists a unique geodesic $\gamma_{b, c}$ joining them. A Finsler structure is given in $C_{a}$ which allows us to define the length of a curve $\gamma \subseteq C_{a}, L(\gamma)$. It holds that the geodesic $\gamma_{b, c}$ has minimal length among all curves with endpoints $b$ and $c$. Finally if $d(b, c)$ is the geodesic distance between $b$ and $c$, then $d$ is a complete distance in $C_{a}$ and $d(b, c)=d_{T}(b, c)=L\left(\gamma_{b, c}\right)$.

\section{Preliminaries}

Let $\mathcal{H}$ be a Hilbert space, $L(\mathcal{H})$ the algebra of linear bounded operators in $\mathcal{H}$, $L(\mathcal{H})^{s}$ the subset of selfadjoint operators and $L(\mathcal{H})^{+}$the cone of positive operators of $L(\mathcal{H})$. Denote by $G L(\mathcal{H})$ the group of invertible operators of $L(\mathcal{H})$ and by $G L(\mathcal{H})^{s}=L(\mathcal{H})^{s} \cap G L(\mathcal{H})$ the set of invertible selfadjoint operators. Let $\mathcal{U}$ be the subgroup of $G L(\mathcal{H})$ of unitary operators and $\mathcal{P}$ the subset of reflections of $\mathcal{U}$; i.e., $\mathcal{P}=\left\{u \in L(\mathcal{H}): u=u^{-1}=u^{*}\right\}$. The range and the nullspace of $a \in L(\mathcal{H})$ are $R(a)$ and $N(a)$, respectively. If $\mathcal{S}$ is a closed subspace of $\mathcal{H}, p_{\mathcal{S}}$ denotes the orthogonal projection onto $\mathcal{S}$. Observe that $p_{\mathcal{S}}$ induces a matrix decomposition as follows: if $p=p_{\mathcal{S}}$ each $a \in L(\mathcal{H})$ can be written as $a=\left(\begin{array}{ll}a_{11} & a_{12} \\ a_{21} & a_{22}\end{array}\right)$, where $a_{11}=\operatorname{pap}_{\left.\right|_{\mathcal{S}}} \in L(\mathcal{S}), a_{12}=p a(1-p)_{\left.\right|_{\mathcal{S}} \perp} \in L\left(\mathcal{S}^{\perp}, \mathcal{S}\right), a_{21}=(1-p) a p_{\left.\right|_{\mathcal{S}}} \in L\left(\mathcal{S}, \mathcal{S}^{\perp}\right)$ and $a_{22}=(1-p) a(1-p)_{\left.\right|_{\mathcal{S}}} \in L\left(\mathcal{S}^{\perp}\right)$.

Every operator $a \in L(\mathcal{H})$ admits a polar decomposition $a=v|a|$ where $|a|=$ $\left(a^{*} a\right)^{1 / 2}$ is positive and $v$ is a partial isometry from $N(a)^{\perp}$ onto $\overline{R(a)}$. Observe that, in general, this decomposition is not unique. To fix the isometric part, define $v_{a}$ as the partial isometry verifying that $v_{a}: N(a)^{\perp} \rightarrow \overline{R(a)}$ is an isometry and $N\left(v_{a}\right)=N(a)$. If $a$ is selfadjoint, the isometric part of the polar decomposition can be defined to obtain a reflection: in this case $R(a)^{\perp}=N(a)$ so that if $u_{a}=$ $v_{a}+p_{N(a)}$, it is easy to see that $u_{a} \in \mathcal{P}$ and $a=u_{a}|a|=|a| u_{a}$. Notice that $u_{\left.a\right|_{N(a)}}=i d_{\left.\right|_{N(a)}}$ and if $p_{a}=p_{\overline{R(a)}}, u_{a} p_{a}=v_{a}$. If $q_{a}=\frac{u_{a}+1}{2}$, then $q_{a}$ is an orthogonal projection and $u_{a} q_{a}=q_{a}$.

From now on, given $a \in L(\mathcal{H})^{s}$ denote by $a=u_{a}|a|$ the polar decomposition of $a$ with $u_{a} \in \mathcal{P}$ and $a=v_{a}|a|$ the polar decomposition of $a$ with $v_{a}$ the partial isometry defined before.

Lemma 2.1. Consider $a \in L(\mathcal{H})^{s}$ with polar decomposition $a=u_{a}|a|$. Then $a$ admits a unique decomposition $a=a_{1}-a_{2}$ such that $a_{1}, a_{2}$ are (semidefinite) positive and $a_{1} a_{2}=0$. Moreover $a_{1}=\frac{|a|+a}{2}=a q_{a}$ and $a_{2}=\frac{|a|-a}{2}=-a\left(1-q_{a}\right)$, where $q_{a}=\frac{u_{a}+1}{2}$.

Proof. Straightforward.

Given $a \in L(\mathcal{H})^{s}$ we will refer to the decomposition of Lemma 2.1 as the positive decomposition of $a$. 
Consider $a \in L(\mathcal{H})^{s}$ with positive decomposition $a=a_{1}-a_{2}$; then $|a|=a_{1}+a_{2}$. If $p_{c}=p_{\overline{R(c)}}$, then $v_{a}=p_{a_{1}}-p_{a_{2}}, u_{a}=p_{a_{1}}-p_{a_{2}}+p_{N(a)}$ and $p_{a}=p_{a_{1}}+p_{a_{2}}$. Notice also that $R\left(a_{1}\right)$ and $R\left(a_{2}\right)$ are closed if and only if $R(a)$ is closed. Finally observe that $R\left(q_{a}\right)=\overline{R\left(a_{1}\right)} \oplus N(a)$ and $N\left(q_{a}\right)=\overline{R\left(a_{2}\right)}$.

Thompson metric for positive operators. Every closed convex cone $K$ of a real Banach space $E$ defines an order relation: if $x, y \in E, x \leq y$ if and only if $y-x \in K$. Suppose that $K$ is normal; i.e., there exists a constant $r>0$ such that $\|x\| \leq r\|y\|$ if $0 \leq x \leq y$. Consider the following equivalence relation in $K$ : $x \sim y$ if there exist constants $r, s>0$ such that $x \leq r y$ and $y \leq s x$. The Thompson component of $x \in E, C_{x}$, is the equivalence class of $x$. Thompson [13] proved that

$$
d_{T}(x, y)=\log \max \{\inf \{r>0: x \leq r y\}, \inf \{s>0: y \leq s x\}\}
$$

defines a complete distance on each component of $K$. The distance $d_{T}$ is known as the part metric or Thompson metric. In the papers by R. Nussbaum [10] and [11] some applications and examples of this metric can be found.

Observe that if $\mathcal{H}$ is a Hilbert space, then $L(\mathcal{H})^{+}$is a closed normal convex cone and the above relation can be considered in $L(\mathcal{H})^{+}$.

The following proposition shows that the components $C_{a}, a \in L(\mathcal{H})^{+}$, are parametrized by the ranges of $a^{1 / 2}$.

Proposition 2.2. Consider $a, b \in L(\mathcal{H})^{+}$; then $b \in C_{a}$ if and only if $R\left(a^{1 / 2}\right)=$ $R\left(b^{1 / 2}\right)$.

Proof. See Corollary 3.3 of [2].

Each $C_{a}$ admits a structure of homogeneous space of an appropriated group. In particular, if $a \in L(\mathcal{H})^{+}$has closed range, then $C_{a}$ identifies with $G L(R(a))^{+}$. There is a natural connection on the associated tangent bundle, $T C_{a}$, and given $b, c \in C_{a}$ there exists a unique geodesic in $C_{a}$ joining $b$ to $c$, namely, $\gamma_{b, c}(t)=$ $b^{1 / 2}\left(b^{-1 / 2} c b^{-1 / 2}\right)^{t} b^{1 / 2}, t \in[0,1]$. Also the geodesic distance, $d(b, c)=\inf \{L(\gamma)\}$ (where the infimun is taken over all smooth curves in $C_{a}$, joining $b$ to $c$ and $L(\gamma)$ is the length of $\gamma$ ), coincides with the Thompson metric, $d_{T}$, on each component. Moreover, if $b, c \in C_{a}$ it holds that

$$
d_{T}(b, c)=L\left(\gamma_{b, c}\right)=\left\|\log \left(b^{-1 / 2} c b^{-1 / 2}\right)\right\|,
$$

where $b^{-1 / 2}=\left(b^{1 / 2}\right)^{\dagger}{ }_{R(a)}$ and $\left(b^{1 / 2}\right)^{\dagger}$ denotes the Moore-Penrose pseudoinverse of $b^{1 / 2}$. See [2] and [3] for a complete exposition of these facts.

\section{Homogeneous structure of $G L(\mathcal{H})^{s}$}

In what follows we recall some results about the geometry of the set of invertible selfadjoint operators, $G L(\mathcal{H})^{s}$, that can be found in [5]. The main result of this section is a characterization of the orbit of an invertible selfadjoint operator given by the action of $G L(\mathcal{H})$ in terms of the polar decomposition of its elements.

Given $a \in G L(\mathcal{H})^{s}$ define in $\mathcal{H}$ the following sesquilinear indefinite form:

$$
\langle x, y\rangle_{a}=\langle a x, y\rangle, \quad x, y \in \mathcal{H} .
$$

The adjoint of $u \in L(\mathcal{H})$ with respect to $\langle,\rangle_{a}$ or the $a$-adjoint of $u$ is $u^{*_{a}}=a^{-1} u^{*} a$. It is easy to see that the group $\mathcal{U}_{a}$ of $a$-unitary elements consists on the operators $u \in G L(\mathcal{H})$ such that $u^{-1}=a^{-1} u^{*} a$. 
Consider the following action of $G L(\mathcal{H})$ on $L(\mathcal{H})^{s}$ :

$L: G L(\mathcal{H}) \times L(\mathcal{H})^{s} \rightarrow L(\mathcal{H})^{s}, L_{g} a=g a g^{*}, \quad$ for $a \in L(\mathcal{H})^{s}$ and $g \in G L(\mathcal{H})$.

Given $a \in L(\mathcal{H})^{s}$, the orbit of $a$ corresponding to the action $L$ is the set

$$
\mathcal{O}_{a}=\left\{g a g^{*}: g \in G L(\mathcal{H})\right\} .
$$

Observe that if $a$ is invertible, then $\mathcal{O}_{a} \subseteq G L(\mathcal{H})^{s}$, so that $L: G L(\mathcal{H}) \times$ $G L(\mathcal{H})^{s} \rightarrow G L(\mathcal{H})^{s}$. The action $L$ is locally transitive in $G L(\mathcal{H})^{s}$; see [5].

The isotropy group of $a \in G L(\mathcal{H})^{s}, I_{a}$, is the group of operators $g \in G L(\mathcal{H})$ such that $L_{g} a=a$; i.e., $I_{a}=\left\{g \in G L(\mathcal{H}): g a g^{*}=a\right\}$. Then $I_{a}=\mathcal{U}_{a^{-1}}$.

Proposition 3.1. Given $a \in G L(\mathcal{H})^{s}$, consider the map $f_{a}: G L(\mathcal{H}) \rightarrow \mathcal{O}_{a}$, $f_{a}(g)=L_{g} a=g_{a} g^{*}$. Then $\left(G L(\mathcal{H}), \mathcal{O}_{a}, f_{a}\right)$ is a principal fibre bundle with structural group $I_{a}$.

Proof. See Proposition 1.1 of [5].

The next result shows that the orbit of $a \in G L(\mathcal{H})^{s}$ is the orbit of its unitary part.

Lemma 3.2. Consider $a \in G L(\mathcal{H})^{s}$ with polar decomposition $a=u_{a}|a|, u_{a} \in \mathcal{P}$; then $\mathcal{O}_{a}=\mathcal{O}_{u_{a}}$.

Proof. Since $a$ is selfadjoint, $a=u_{a}|a|=|a|^{1 / 2} u_{a}|a|^{1 / 2}$. Then $a \in \mathcal{O}_{u_{a}}$ so that $\mathcal{O}_{a}=\mathcal{O}_{u_{a}}$.

Given $w \in \mathcal{U}$ the unitary orbit of $w$ is the set $\mathcal{U O}_{w}=\left\{u w u^{*}: u \in \mathcal{U}\right\}$. The following theorem relates the orbits $\mathcal{O}_{a}$ and $\mathcal{U} \mathcal{O}_{u_{a}}$.

Theorem 3.3. Consider $a, b \in G L(\mathcal{H})^{s}$ with polar decompositions $a=u_{a}|a|, b=$ $u_{b}|b|$ and positive decompositions $a=a_{1}-a_{2}, b=b_{1}-b_{2}$. Then the following conditions are equivalent:

(1) $b \in \mathcal{O}_{a}$,

(2) $u_{b} \in \mathcal{U} \mathcal{O}_{u_{a}}$,

(3) $\operatorname{dim} R\left(a_{i}\right)=\operatorname{dim} R\left(b_{i}\right)$, for $i=1,2$.

Proof. (1) $\rightarrow(2)$ : Consider $b \in \mathcal{O}_{a}$; then, by Lemma 3.2, $u_{b} \in \mathcal{O}_{u_{a}}$, so that there exists $g \in G L(\mathcal{H})$ such that $u_{b}=g u_{a} g^{*}$. Therefore $u_{b}=g u_{a} g^{*}=\left(g^{*}\right)^{-1} u_{a} g^{-1}$ or $g^{*} g u_{a} g^{*} g=u_{a}$. Consider $\lambda=g^{*} g$; then $\lambda u_{a} \lambda=u_{a}$ or $\lambda^{-1}=u_{a} \lambda u_{a}$. Since $\lambda>0$ and $u_{a} \in \mathcal{P}$, it follows that $\lambda^{-1 / 2}=u_{a} \lambda^{1 / 2} u_{a}$ or $\lambda^{-1 / 2} u_{a}=u_{a} \lambda^{1 / 2}$. If $g=w \lambda^{1 / 2}$ is the polar decomposition of $g$ with $w \in \mathcal{U}$, we get that $u_{b}=w \lambda^{1 / 2} u_{a} \lambda^{1 / 2} w^{*}=$ $w u_{a} w^{*}$, so that $u_{b} \in \mathcal{U} \mathcal{O}_{u_{a}}$.

$(2) \rightarrow(3)$ : Consider $u_{b} \in \mathcal{U} \mathcal{O}_{u_{a}}$; then there exists $u \in \mathcal{U}$ such that $u_{b}=u u_{a} u^{*}$. Therefore $p_{b_{1}}-p_{b_{2}}=u_{b}=u u_{a} u^{*}=u p_{a_{1}} u^{*}-u p_{a_{2}} u^{*}$. It follows from Lemma 2.1 that $p_{b_{i}}=u p_{a_{i}} u^{*}$ so that $\operatorname{dim} R\left(a_{i}\right)=\operatorname{dim} R\left(b_{i}\right)$, for $i=1,2$.

$(3) \rightarrow(1)$ : Since $\operatorname{dim} R\left(b_{i}\right)=\operatorname{dim} R\left(a_{i}\right)$ for $i=1,2$, there exists a partial isometry $u_{i}$ from $\mathcal{H}$ onto $R\left(b_{i}\right)$ with $N\left(u_{i}\right)=R\left(a_{i}\right)^{\perp}$. Consider $u=u_{1}+u_{2}$; then $u \in \mathcal{U}$ because $R\left(a_{1}\right) \oplus R\left(a_{2}\right)=\mathcal{H}$ and $R\left(a_{1}\right)^{\perp}=R\left(a_{2}\right)$. Also $u u_{a} u^{*}=$ $u\left(p_{a_{1}}-p_{a_{2}}\right) u^{*}=p_{b_{1}}-p_{b_{2}}=u_{b}$, so that $u_{b} \in \mathcal{O}_{u_{a}}$, or equivalently $b \in \mathcal{O}_{a}$.

Consider the map

$$
\pi: G L(\mathcal{H})^{s} \rightarrow \mathcal{P}, \quad \pi(a)=u_{a}, \quad \text { where } a \in G L(\mathcal{H})^{s}, a=u_{a}|a| .
$$


It follows from the definition of $\pi$ that the fibre of $u \in \mathcal{P}, \pi^{-1}(\{u\})$, is the subset of $G L(\mathcal{H})^{s}$ of elements $a=u_{a}|a|$ such that $u_{a}=u$; i.e., $\pi^{-1}(\{u\})=\left\{a \in G L(\mathcal{H})^{s}\right.$ : $a=u \alpha, \alpha>0\}$. In particular, the fibre of 1 is $G L(\mathcal{H})^{+}$.

The following lemma is similar to Proposition 4.1 of [5].

Lemma 3.4. Consider $u \in \mathcal{P}$; then the following conditions are equivalent:

(1) $a \in \pi^{-1}(\{u\})$,

(2) $a=a^{*}, a u>0$

(3) $a u=u a, a u>0$.

Proof. Straightforward.

\section{Equivalence Relations in $L(\mathcal{H})^{s}$}

From now on, given $c \in L(\mathcal{H})^{s}, c=c_{1}-c_{2}$ denotes the positive decomposition of $c$ defined in the Preliminaries and $p_{c}=p_{\overline{R(c)}}$. Recall that $c=u_{c}|c|=v_{c}|c|$ denotes the polar decompositions of $c$ with $u_{c} \in \mathcal{P}$ and $v_{c}$ a partial isometry.

Given $v_{a}$ and $v_{b}$ partial isometries, $v_{a}$ is unitarily equivalent to $v_{b}$ if there exits $u \in \mathcal{U}$ such that $v_{b}=u v_{a} u^{*}$. The unitary orbit of $v_{a}$ is the set $\mathcal{U} \mathcal{O}_{v_{a}}=\left\{u v_{a} u^{*}\right.$ : $u \in \mathcal{U}\}$.

Given a reflection $v \in \mathcal{P}$, consider the indefinite sesquilinear form given by

$$
\langle x, y\rangle_{v}=\langle v x, y\rangle, \quad x, y \in \mathcal{H},
$$

and define in $L(\mathcal{H})$ the following order: given $a, b \in L(\mathcal{H})$, then $a \leq_{v} b$ if and only if $\langle(b-a) x, x\rangle_{v} \geq 0 \forall x \in \mathcal{H}$, or equivalently, $v(b-a) \geq 0$.

In what follows we define three equivalence relations in $L(\mathcal{H})^{s}$ that extend the relation in $L(\mathcal{H})^{+}$discussed in the Preliminaries. Consider $a, b \in L(\mathcal{H})^{s}$, then

(1) $a \sim b$, if there exist $\alpha, \beta>0$ such that $a \leq_{u_{a}} \alpha b$ and $b \leq_{u_{b}} \beta b$.

(2) $a \sim_{1} b$, if there exist $\alpha, \beta>0$ such that $|a| \leq \alpha|b|,|b| \leq \beta|a|$ and $v_{a}$ is unitarily equivalent to $v_{b}$.

(3) $a \sim_{2} b$, if there exist $\alpha, \beta>0$ such that $|a| \leq \alpha|b|$ and $|b| \leq \beta|a|$.

It is not difficult to see that $\sim_{1}$ and $\sim_{2}$ are equivalence relations.

To see that $\sim$ is an equivalence relation we need the following lemma.

Lemma 4.1. Consider $a, b \in L(\mathcal{H})^{s}$ with polar decompositions $a=u_{a}|a|, b=u_{b}|b|$. If $a \sim b$, then $u_{a}=u_{b}$.

Proof. If $a \sim b$, then there exist $\alpha, \beta>0$ such that $u_{a}(\alpha b-a) \geq 0$ and $u_{b}(\beta a-b) \geq 0$. Then $c=\alpha u_{a} b \geq|a| \geq 0$, and $b=\alpha^{-1} u_{a} c$. Since the polar decomposition is unique in $\overline{R(b)}$, it follows that $u_{a}=u_{b}$ in $\overline{R(b)}$. It remains to prove that $u_{a}=u_{b}$ in $N(b)$. In fact $N(a)=N(b)$ : if $x \in N(b)$, then $0 \leq\langle(c-|a|) x, x\rangle=-\langle|a| x, x\rangle$ so that $|a| x=0$. Hence, $x \in N(|a|)=N(a)$ and $N(b) \subseteq N(a)$. In the same way, using the fact that $u_{b}(\beta a-b) \geq 0$ it follows that $N(a) \subseteq N(b)$. Then $u_{a}=u_{b}$ in $N(a)$ by definition (see the Preliminaries).

Corollary 4.2. The relation $\sim$ is an equivalence relation.

Proof. Apply Lemma 4.1.

Denote by $C_{a}, C_{a}^{1}$ and $C_{a}^{2}$ the equivalence classes or components of $a$ corresponding to $\sim, \sim_{1}$ and $\sim_{2}$, respectively.

As a corollary of Lemma 4.1 we obtain a characterization of the component $C_{a}$ of $a$ in terms of its polar decomposition. 
Corollary 4.3. Consider $a \in L(\mathcal{H})^{s}$, with polar decomposition $a=u_{a}|a|$. Then

$$
C_{a}=\left\{b \in L(\mathcal{H})^{s}: R\left(|b|^{1 / 2}\right)=R\left(|a|^{1 / 2}\right) \text { and } u_{b}=u_{a}\right\} .
$$

Proof. Consider $b \in C_{a}$ with polar decomposition $b=|b| u_{b}$; then by Lemma 4.1, it follows that $u_{a}=u_{b}$. Also there exist $\alpha, \beta>0$ such that $u_{a}(\alpha b-a) \geq 0$ and $u_{b}(\beta a-b) \geq 0$ so that $|a| \leq \alpha|b|$ and $|b| \leq \beta|a|$. Therefore $|a| \sim|b|$, and by Proposition 2.2, this is equivalent to $R\left(|b|^{1 / 2}\right)=R\left(|a|^{1 / 2}\right)$. The converse is similar.

Corollary 4.4. Consider $a=v_{a}|a| \in L(\mathcal{H})^{s}$ with closed range; then $C_{a}=C_{v_{a}}$.

Proof. Since $a$ has closed range, $b \in C_{a}$ if and only if $R(b)=R(a)$ and $u_{b}=u_{a}$, or equivalently, $R(b)=R(a)$ and $v_{b}=v_{a}$. But observe that, in this case, $R\left(v_{a}\right)=$ $R(a)$. Therefore $v_{a} \in C_{a}$.

It follows from Proposition 2.2 that $C_{a}^{1}=\left\{b \in L(\mathcal{H})^{s}: R\left(|b|^{1 / 2}\right)=R\left(|a|^{1 / 2}\right)\right.$ and $\left.v_{b} \in \mathcal{U} \mathcal{O}_{v_{a}}\right\}$ and $C_{a}^{2}=\left\{b \in L(\mathcal{H})^{s}: R\left(|b|^{1 / 2}\right)=R\left(|a|^{1 / 2}\right)\right\}$.

Given $a \in L(\mathcal{H})^{s}$, denote by $\tilde{a}=\left.a\right|_{R(a)}$. If $a$ has closed range, then $\tilde{a} \in$ $G L(R(a))^{s}$. Consider the orbit of $\tilde{a}$ in $R(a)$; i.e., $\mathcal{O}_{\tilde{a}}=\left\{g \tilde{a} g^{*}: g \in G L(R(a))\right\}$. Observe that it follows from Theorem 3.3 that if $a \in G L(\mathcal{H})^{s}$, then $\mathcal{O}_{a}=C_{a}^{1}$. More generally,

Proposition 4.5. Let $a \in L(\mathcal{H})^{s}$ be a closed range operator and $b \in L(\mathcal{H})^{s}$. Then the following conditions are equivalent:

(1) $b \in C_{a}^{1}$,

(2) $R(b)=R(a)$ and $\operatorname{dim} R\left(b_{i}\right)=\operatorname{dim} R\left(a_{i}\right)$, for $i=1,2$,

(3) $\tilde{b} \in \mathcal{O}_{\tilde{a}}$.

Proof. (1) $\rightarrow$ (2): If $b \in C_{a}^{1}$, then $R\left(|b|^{1 / 2}\right)=R\left(|a|^{1 / 2}\right)$. Since $a$ has closed range $R(a)=R\left(|a|^{1 / 2}\right)$ so that $R\left(|b|^{1 / 2}\right)$ is closed. Then $R\left(|b|^{1 / 2}\right)=|b|^{1 / 2}\left(N(b)^{\perp}\right)=$ $|b|^{1 / 2}\left(R\left(|b|^{1 / 2}\right)\right)=R(b)$. Therefore $R(b)=R(a)$. Also $v_{b} \in \mathcal{U} \mathcal{O}_{v_{a}}$, so that $v_{b}=$ $u v_{a} u^{*}$ for $u \in \mathcal{U}$. In the same way as in the proof of $(2) \rightarrow(3)$ of Theorem 3.3 it can be proved that $\operatorname{dim} R\left(b_{i}\right)=\operatorname{dim} R\left(a_{i}\right)$ for $i=1,2$.

$(2) \rightarrow(3)$ : Notice that $\tilde{b} \in G L(R(a))$ because $R(b)=R(a)$. Then apply Theorem 3.3 to $G L(R(a))^{s}$.

$(3) \rightarrow(1):$ Consider $b \in L(\mathcal{H})^{s}$ such that $\tilde{b}=g \tilde{a} g^{*}$ with $g \in G L(R(a))$. Then $R(b)=R(\tilde{b})=R(a)$. By Theorem 3.3, $u_{\tilde{b}} \in \mathcal{U} \mathcal{O}_{u_{\tilde{a}}}$. If $c \in L(\mathcal{H})^{s}$ it holds that $u_{\tilde{c}}=\tilde{v}_{c}$, therefore $\tilde{v_{b}} \in \mathcal{U} \mathcal{O}_{\tilde{v_{a}}}$; i.e., there exists $u \in \mathcal{U}(R(a))$ such that $\tilde{v_{b}}=u \tilde{v_{a}} u^{*}$. If $w=u+p_{N(a)}$, then $w \in \mathcal{U}$ and $v_{b}=w v_{a} w^{*}$. Therefore $b \in C_{a}^{1}$.

Remark 4.6. In Section 3 we defined the map

$$
\pi: G L(\mathcal{H})^{s} \rightarrow \mathcal{P}, \pi(b)=u_{b}, \quad \text { for } b \in G L(\mathcal{H})^{s} \text { with } b=u_{b}|b| .
$$

From the definitions and properties studied in Section 3, if $u \in \mathcal{P}$, it holds that

$$
\pi^{-1}(\{u\}) \subseteq \mathcal{O}_{u} \subseteq G L(\mathcal{H})^{s} .
$$

Notice that $\pi^{-1}(\{u\})=C_{u}, \mathcal{O}_{u}=C_{u}^{1}$ and $G L(\mathcal{H})^{s}=C_{u}^{2}$. Analogously, if $a$ is a closed range operator, consider the map

$$
\pi_{a}: G L(R(a))^{s} \rightarrow \mathcal{P}(R(a)), \quad \pi(b)=u_{b},
$$


where $G L(R(a))^{s}$ is the set of invertible selfadjoint operators in $R(a), \mathcal{P}(R(a))$ is the subset of reflections in $R(a)$ and $b=u_{b}|b|$ is the polar decomposition of $b \in G L(R(a))^{s}$. Then, by Corollary 4.3 and Proposition 4.5, we can identify

$$
\pi_{a}^{-1}\left(\left\{\tilde{u}_{a}\right\}\right) \approx C_{a}, \quad \mathcal{O}_{\tilde{a}} \approx C_{a}^{1}, \quad G L(R(a))^{s} \approx C_{a}^{2} .
$$

More generally, given $a \in L(\mathcal{H})^{s}$, it holds that $C_{a} \subset C_{a}^{1} \subset C_{a}^{2}$. Therefore $\sim, \sim_{1}$ and $\sim_{2}$ extend to $L(\mathcal{H})^{s}$ the relation in $L(\mathcal{H})^{+}$described in the Preliminaries. In fact, if $a \in L(\mathcal{H})^{+}$and $b \in L(\mathcal{H})^{s}$ is such that $b \in C_{a}$, then $1=u_{a}=u_{b}$ so that $b \in L(\mathcal{H})^{+}$and there exist $\alpha, \beta>0$ such that $a \leq \alpha b$ and $b \leq \beta a$. Therefore, when $a$ is positive, $C_{a}$ coincides with the Thompson component of $a$.

\section{The Thompson Components of $L(\mathcal{H})^{s}$}

The next theorem proves that given $a \in L(\mathcal{H})^{s}, C_{a}$ is homeomorphic to the product of two Thompson components of positive operators. This characterization naturally leads to the definition of a metric on $C_{a}$ that extends the Thompson metric. From now on we will refer to $C_{a}$ as the Thompson component of $a$.

Theorem 5.1. Consider $a \in L(\mathcal{H})^{s}$ with positive decomposition $a=a_{1}-a_{2}$. Then $C_{a}$ is homeomorphic to $C_{a_{1}} \times C_{a_{2}}$.

Proof. Given $b \in L(\mathcal{H})^{s}$ with positive decomposition $b=b_{1}-b_{2}$, define $\psi: L(\mathcal{H})^{s} \rightarrow$ $L(\mathcal{H})^{+} \times L(\mathcal{H})^{+}, \psi(b)=\left(b_{1}, b_{2}\right)$. The map $\psi$ is well defined because, by Lemma 2.1 , the decomposition is unique; also $\psi$ is injective. If $b \in C_{a}, u_{a}=u_{b}$ and there exist $\alpha, \beta>0$ such that $(\alpha b-a) u_{a} \geq 0$ and $(\beta a-b) u_{a} \geq 0$, then $q_{a}(\alpha b-a) u_{a} q_{a}=$ $q_{a}(\alpha b-a) q_{a} \geq 0$, with $q_{a}=\frac{u_{a}+1}{2}$. Since $b q_{a}=b_{1}$ and $a q_{a}=a_{1}$, it follows that $\alpha b_{1}-a_{1} \geq 0$. Similarly, $\beta a_{1} \geq b_{1}$. Hence $b_{1} \in C_{a_{1}}$. In the same way, $b_{2} \in C_{a_{2}}$.

To see that $\psi$ is surjective consider $\left(b_{1}, b_{2}\right) \in C_{a_{1}} \times C_{a_{2}}$; then $b=b_{1}-b_{2} \in L(\mathcal{H})^{s}$, $b_{1}, b_{2} \geq 0$ and $b_{1} b_{2}=0$. Hence, by Lemma 2.1, if $b=b_{1}-b_{2}$, then $b \in L(\mathcal{H})^{s}$. Moreover $b \in C_{a}$ : since $b_{i} \in C_{a_{i}}$ there exist $\alpha_{i}>0$ such that $a_{i} \leq \alpha_{i} b_{i}$ for $i=1,2$. Hence $|a| \leq \alpha_{1} b_{1}+\alpha_{2} b_{2} \leq \alpha^{\prime}\left(b_{1}+b_{2}\right) \leq \alpha^{\prime}|b|$, where $\alpha^{\prime}=\max \left\{\alpha_{1}, \alpha_{2}\right\}$. In a similar way, there exists $\beta^{\prime}>0$ such that $|b| \leq \beta^{\prime}|a|$. Therefore $|b| \in C_{|a|}$. It remains to prove that $u_{a}=u_{b}$, or equivalently that $q_{a}=q_{b}$. Since $|b| \in C_{|a|}$, then $N(a)=N(b)$. Also $\overline{R\left(a_{1}\right)}=\overline{R\left(b_{1}\right)}$ because $b_{1} \in C_{a_{1}}$. Then $R\left(q_{a}\right)=\overline{R\left(a_{1}\right)} \oplus N(a)$ (see the Preliminaries). Therefore $R\left(q_{a}\right)=R\left(q_{b}\right)$ so that $q_{a}=q_{b}$, because $q_{a}$ and $q_{b}$ are orthogonal projectors.

Remark 5.2. Consider $a \in L(\mathcal{H})^{s}$ with positive decomposition $a=a_{1}-a_{2}$. Then, from the proof of Theorem 5.1, the component of $a$ can be written as

$$
C_{a}=\left\{b_{1}-b_{2} \in L(\mathcal{H})^{s}: b_{i} \in C_{a_{i}}, i=1,2\right\} .
$$

Consider $a, b, c \in L(\mathcal{H})^{s}$ with positive decompositions $a=a_{1}-a_{2}, b=b_{1}-b_{2}$ and $c=c_{1}-c_{2}$. If $b, c \in C_{a}$, define

$$
\left.d(b, c)=\max \left\{d_{T}\left(b_{i}, c_{i}\right), i=1,2\right)\right\},
$$

where $d_{T}\left(b_{i}, c_{i}\right)=\log \max \left\{\inf \left\{r>0: b_{i} \leq r c_{i}\right\}, \inf \left\{s>0: c_{i} \leq s b_{i}\right\}\right\}$ for $i=1,2$ is the Thompson metric for positive operators considered in the Preliminaries.

Observe that $d$ is well defined because of the uniqueness of the positive decomposition of selfadjoint operators and the fact that $b_{i}, c_{i} \in C_{a_{i}}, i=1,2$, where $d_{T}$ is defined. It is easy to see that $d$ defines a complete metric on $C_{a}$. Furthermore 
observe that the metric $d$ coincides with $d_{T}$ on each positive component. From now on, denote by $d=d_{T}$ the Thompson metric on $C_{a}$.

If $c \in L(\mathcal{H})^{s}$ has closed range, denote by $c^{-1}=\left(c_{\left.\right|_{R(c)}}\right)^{-1}=\left.c^{\dagger}\right|_{R(c)}$, where $c^{\dagger}$ is the Moore-Penrose pseudoinverse of $c$. Observe that $c^{-1} \in G L(R(c))$.

Proposition 5.3. Given $a \in L(\mathcal{H})^{s}$ with closed range, consider $b, c \in C_{a}$ with positive decompositions $b=b_{1}-b_{2}$ and $c=c_{1}-c_{2}$; then

$$
d_{T}(b, c)=\left\|\log \left[\left(\left(b_{1}^{1 / 2}\right)^{\dagger} c_{1}\left(b_{1}^{1 / 2}\right)^{\dagger}+\left(b_{2}^{1 / 2}\right)^{\dagger} c_{2}\left(b_{2}^{1 / 2}\right)^{\dagger}\right)_{\left.\right|_{R(b)}}\right]\right\| .
$$

Proof. Since $b, c \in C_{a}$ and $a=a_{1}-a_{2}$ is the positive decomposition of $a$, then for $i=1,2, b_{i}, c_{i} \in C_{a_{i}}$. Since $d_{i}=b_{i}^{-1 / 2} c_{i} b_{i}^{-1 / 2} \in G L\left(R\left(b_{i}\right)\right)^{+}$, then, by (2.1),

$$
\begin{aligned}
d_{T}(b, c) & =\max \left\{\left\|\log d_{i}\right\|, i=1,2\right\} \\
& =\left\|\left(\begin{array}{cc}
\log d_{1} & 0 \\
0 & \log d_{2}
\end{array}\right)\right\|=\left\|\log \left(\begin{array}{cc}
d_{1} & 0 \\
0 & d_{2}
\end{array}\right)\right\| \\
& =\left\|\log \left[\left(\left(b_{1}^{1 / 2}\right)^{\dagger} c_{1}\left(b_{1}^{1 / 2}\right)^{\dagger}+\left(b_{2}^{1 / 2}\right)^{\dagger} c_{2}\left(b_{2}^{1 / 2}\right)^{\dagger}\right)_{\left.\right|_{R(b)}}\right]\right\| .
\end{aligned}
$$

Corollary 5.4. Consider $a \in L(\mathcal{H})^{s}$ with closed range. If $b, c \in C_{a}$, then

$$
d_{T}(b, c)=d_{T}(|b|,|c|) .
$$

Proof. If $b, c \in C_{a}$, then $|b|,|c| \in C_{|a|}$ and, by Theorem 3.11 of [2], it holds that $d_{T}(|b|,|c|)=\left\|\log \left(|b|^{-1 / 2}|c||b|^{-1 / 2}\right)\right\|$. On the other hand, it is easy to see that $\left(|b|^{1 / 2}\right)^{\dagger}|c|\left(|b|^{1 / 2}\right)^{\dagger}=\left(b_{1}^{1 / 2}\right)^{\dagger} c_{1}\left(b_{1}^{1 / 2}\right)^{\dagger}+\left(b_{2}^{1 / 2}\right)^{\dagger} c_{2}\left(b_{2}^{1 / 2}\right)^{\dagger}$ where $b=b_{1}-b_{2}$ and $c=$ $c_{1}-c_{2}$ are the positive decompositions of $b$ and $c$, respectively. Then, applying Proposition 5.3, it follows that $d_{T}(b, c)=d_{T}(|b|,|c|)$.

As in the positive case, the metrical structure of $\left(C_{a}, d_{T}\right)$ is related to a differential structure. Consider $a \in L(\mathcal{H})^{s}$ with closed range and positive decomposition $a=a_{1}-a_{2}$. Then $C_{a_{i}}$ identifies with $G L\left(R\left(a_{i}\right)\right)^{+}$for $i=1,2$ (see [2], [3]) and, as it was pointed out in the Preliminaries, $C_{a_{i}}$ is a homogeneous space of $G L\left(R\left(a_{i}\right)\right)$, $i=1,2$. The reader is referred to [3] and [9] in order to have a full description of this structure. Then, since $C_{a}$ is homeomorphic to $C_{a_{1}} \times C_{a_{2}}$ (see Theorem 5.1), $C_{a}$ admits a natural structure of homogeneous space of $G L\left(R\left(a_{1}\right)\right) \times G L\left(R\left(a_{2}\right)\right)$.

A natural connection can be defined in $C_{a}$, which induces the concept of a parallel field along a curve. A curve $\gamma \subseteq C_{a}$ is a geodesic if $\dot{\gamma}$ is parallel along $\gamma$ and, in this case $\gamma$ satisfies $\ddot{\gamma}=\dot{\gamma} \gamma^{\dagger} \dot{\gamma}$ (see [2], [3]).

For $b \in C_{a}$, denote by $\left(T C_{a}\right)_{b}$ the tangent space of $C_{a}$ at $b$. If $X \in\left(T C_{a}\right)_{b}$ it follows from the product structure considered on $C_{a}$, that $X=X_{1} p_{a_{1}}+X_{2} p_{a_{2}}$, where $X_{i} \in L\left(R\left(a_{i}\right)\right)^{s}, i=1,2$.

The unique geodesic $\gamma$ such that $\gamma(0)=b$ and $\dot{\gamma}=X \in\left(T C_{a}\right)_{b}$ is

$$
\gamma(t)=e^{\frac{t}{2} X b^{\dagger}} b e^{\frac{t}{2} b^{\dagger} X} .
$$

It follows easily that $\gamma(t)=\gamma_{1}(t)-\gamma_{2}(t)$ where $\gamma_{i}(t)=e^{\frac{t}{2} X b_{i}{ }^{\dagger}} b_{i} e^{\frac{t}{2} b_{i}{ }^{\dagger} X}$ is the geodesic such that $\gamma_{i}(0)=b_{i}$ and $\dot{\gamma}_{i}(0)=X_{i} p_{a_{i}} \in\left(T C_{a_{i}}\right)_{b_{i}}, i=1,2$.

Given $b, c \in C_{a}$ there is only one geodesic $\gamma_{b, c}$ such that $\gamma_{b, c}(0)=b$ and $\gamma_{b, c}(1)=$ $c$, namely

$$
\gamma_{b, c}(t)=|b|^{1 / 2}\left(|b|^{-1 / 2}|c||b|^{-1 / 2}\right)^{t}|b|^{1 / 2} v_{a}=\gamma_{|b|,|c|}(t) v_{a}
$$


It is easy to see that

$$
\gamma_{b, c}(t)=\gamma_{b_{1}, c_{1}}(t)-\gamma_{b_{2}, c_{2}}(t),
$$

where $\gamma_{b_{i}, c_{i}}(t)=b_{i}^{1 / 2}\left(b_{i}^{-1 / 2} c_{i} b_{i}^{-1 / 2}\right)^{t} b_{i}^{1 / 2}$ is the geodesic in $C_{a_{i}}$ joining $b_{i}$ and $c_{i}$, $i=1,2$.

The norm

$$
\|X\|_{b}=\left\|\left(|b|^{1 / 2}\right)^{\dagger} X\left(|b|^{1 / 2}\right)^{\dagger}\right\|, \quad X \in\left(T C_{a}\right)_{b},
$$

defines a Finsler structure on the tangent bundle $T C_{a}$; i.e., a smooth assignation of a complete norm on each tangent space $\left(T C_{a}\right)_{b}, b \in C_{a}$. If $X=X_{1} p_{a_{1}}+X_{2} p_{a_{2}}$, with $X_{i} \in L\left(R\left(a_{i}\right)\right)^{s}, i=1,2$, it follows that $\|X\|_{b}=\max \left\{\left\|X_{i}\right\|_{b_{i}}, i=1,2\right\}$.

For a $C^{\infty}$ curve $\gamma:[0,1] \rightarrow C_{a}$ define the length of $\gamma$ by

$$
L(\gamma)=\int_{0}^{1}\|\dot{\gamma}(t)\|_{\gamma(t)} d t
$$

Given $\gamma \subseteq C_{a}$ then $L(\gamma)=\max \left\{L\left(\gamma_{1}\right), L\left(\gamma_{2}\right)\right\}$, where $\gamma=\gamma_{1}-\gamma_{2}$ is the positive decomposition of $\gamma$. In fact, $\|\dot{\gamma}(t)\|_{\gamma(t)}=\max \left\{\left\|\dot{\gamma}_{i}(t)\right\|_{\gamma_{i}(t)}, i=1,2\right\}$.

Lemma 5.5. Given $a \in L(\mathcal{H})^{s}$ with closed range, consider $b, c \in C_{a}$. If $\gamma_{b, c}$ is the unique geodesic joining $b$ and $c$, then

$$
L\left(\gamma_{b, c}\right)=L\left(\gamma_{|b|,|c|}\right)=\max \left\{\left\|\log \left(b_{i}^{-1 / 2} c_{i} b_{i}^{-1 / 2}\right)\right\|, i=1,2\right\} .
$$

Proof. The first equality follows from (5.1). From (5.2) we get that $L\left(\gamma_{b, c}\right)=$ $\max \left\{L\left(\gamma_{b_{i}, c_{i}}\right): i=1,2\right\}$ but $L\left(\gamma_{b_{i}, c_{i}}\right)=\left\|\log \left(b_{i}^{-1 / 2} c_{i} b_{i}^{-1 / 2}\right)\right\|$ (see the Preliminaries or Corollary 2.9 of [2]).

Now we show that among all curves in $C_{a}$ joining $b$ and $c$, the geodesic $\gamma_{b, c}$ has minimal length.

Proposition 5.6. Given a closed range operator $a \in L(\mathcal{H})^{s}$, consider $b, c \in C_{a}$. If $\delta:[0,1] \rightarrow C_{a}$ is a $C^{\infty}$ curve such that $\delta(0)=b$ and $\delta(1)=c$, then $L\left(\gamma_{b, c}\right) \leq L(\delta)$.

Proof. Consider a curve $\delta:[0,1] \rightarrow C_{a}, \delta(t)=|\delta(t)| v_{a}$ such that $\delta(0)=b$ and $\delta(1)=c$. If $b=|b| v_{a}, c=|c| v_{a}$, then it holds that $|\delta|:[0,1] \rightarrow C_{|a|}$ is a curve joining $|b|$ with $|c|$. Then, by Theorem 2.10 of [2], it follows that $L\left(\gamma_{|b|,|c|}\right) \leq L(|\delta|)$. But $L\left(\gamma_{|b|,|c|}\right)=L\left(\gamma_{b, c}\right)$ and $L(\delta)=L(|\delta|)$.

Consider $b, c \in C_{a}$; the geodesic distance in $C_{a}$ is defined by

$$
d(b, c)=\inf \left\{L(\gamma): \gamma:[0,1] \rightarrow C_{a}, C^{\infty}, \gamma(0)=b, \gamma(1)=c\right\} .
$$

Corollary 5.7. Given $a \in L(\mathcal{H})^{s}$ with closed range and $b, c \in C_{a}$, then

$$
d(b, c)=L\left(\gamma_{b, c}\right) .
$$

As in the positive case, the Thompson metric coincides with the geodesic distance on each component.

Corollary 5.8. Consider a closed range operator $a \in L(\mathcal{H})^{s}$. If $b, c \in C_{a}$, then

$$
d_{T}(b, c)=d(b, c)=L\left(\gamma_{b, c}\right)
$$

where $\gamma_{b, c}$ is the geodesic in $C_{a}$ joining $b$ and $c$.

Proof. Apply (2.1), Corollary 5.4 and Lemma 5.5. 
Final remarks. The geometrical structure of $C_{a}$ as a homogeneous space, discussed in Section 5 when $a$ has closed range, is still valid in the general case. However the computations are more complicated. The reader can find in [3] these computations in detail when $a$ is a general positive operator. In Section 3 the orbit of an invertible selfadjoint operator was described by means of the polar decomposition of its elements. More generally, it is interesting to study the orbit of a closed range operator $a \in L(\mathcal{H})^{s}$; i.e., the set $\mathcal{O}_{a}=\left\{g a g^{*}, g \in G L(\mathcal{H})\right\}$. The geometrical and metrical structures of $\mathcal{O}_{a}$ were studied in the positive case, in [4], where it was proved that $\mathcal{O}_{a}$ admits a structure of differential manifold, if an appropriated metric is considered.

\section{REFERENCES}

1. Bonsall, F. F. and Duncan, J., Complete Normed Algebras. Springer-Verlag (1973). MR0423029 (54:11013)

2. Corach, G. and Maestripieri, A., Differential and metrical structure of positive operators. Positivity 4 (1999) 297-315. MR1721561 (2001a:58004)

3. Corach, G. and Maestripieri, A., Differential geometry on Thompson's components of positive operators. Rep. Math. Phys. 45 (2000) 23-37. MR1751502 (2001h:58008)

4. Corach, G., Maestripieri, A. and Stojanoff, D., Orbits of positive operators from a differentiable viewpoint. Positivity 8 (2004) 31-48. MR2053574 (2005d:58011)

5. Corach, G., Porta, H. and Recht, L., The geometry of spaces of selfadjoint invertible elements of a $C^{*}$-algebra. Integral Equations Operator Theory 16 (1993) 333-359. MR1209304 (94d:58010)

6. Douglas, R. G., On majorization, factorization and range inclusion of operators in Hilbert space. Proc. Amer. Math. Soc. 17 (1966) 413-416. MR0203464 (34:3315)

7. Fillmore, P. A. and Williams, J. P., On operator ranges. Adv. Math. 7 (1971) 254-281. MR0293441 (45:2518)

8. Kato, Y., An elementary proof of Sz.-Nagy theorem. Math. Japon. 20 (1975) 257-258. MR0405129 (53:8924)

9. Kobayashi, S. and Nomizu, K., Foundations of differential geometry. Interscience, New York, 1969.

10. Nussbaum, R., Hilbert's projective metric and iterated non linear maps. Mem. Amer. Math. Soc. 391 (1988). MR961211 (89m:47046)

11. Nussbaum, R., Finsler structures for the part metric and Hilbert's projective metric and applications to ordinary differential equations. Differential Integral Equations 7 (1994) 16491707. MR1269677 (95b:58010)

12. Rudin, W., Functional Analysis. McGraw-Hill (1973). MR0365062 (51:1315)

13. Thompson, A.C., On certain contraction mappings in a partially ordered vector space. Proc. Amer. Math. Soc. 14 (1963) 438-443. MR0149237 (26:6727)

Instituto Argentino de Matemática, COniCET, SaAvedra 15, 1083 Buenos Aires, ArGENTINA

E-mail address: fongig@fceia.unr.edu.ar

Instituto de Ciencias, Universidad Nacional General Sarmiento, 1613 Los Polvorines, Argentina

E-mail address: amaestri@ungs.edu.ar 